\title{
Clinical Assessment of Anesthesia Caused by Combinational Protocol of Acepromazine-Fentanyl- Ketamine in Dogs
}

\author{
Adel Khodaei Sharabiyani and Mahmoud Tohidi Moghadam* \\ Graduate of Faculty of Veterinary Medicine Islamic Azad University, Iran
}

\begin{abstract}
The purpose of the present study is the clinical assessment of anesthesia by adding fentanyl to ketamine and acepromazine in dogs and the clinical assessment of dogs during this anesthesia. In this study, 12 mixed-breed dogs, that weigh $20-30 \mathrm{~kg}$ and are 2-3 years old, were selected and divided into two test and control groups. Anesthetizing of the test group is done by intramuscular injection of $0.2 \mathrm{mg}$ of kg acepromazine; and intravenous injection of $0.1 \mathrm{mg}$ $\mathrm{kg}$ of fentanyl and intramuscular injection of $20 \mathrm{mg} \mathrm{kg}$ of ketamine after 10 minutes. Anesthetizing of the control group is done by intramuscular injection of $0.2 \mathrm{mg} \mathrm{kg}$ of acepromazine; and intramuscular injection of $20 \mathrm{mg} \mathrm{kg}$ of ketamine after 10 minutes. One hour after anesthetizing both test and control groups, parameters such as: heart rate, respiratory rate, rectal temperature and clinical symptoms such as duration of analgesia and duration of anesthesia were assessed in 10-minute-interval periods. T independent test, ANOVA, and Tukey Post Hoc were used for the statistical analysis of the data. As the results show, the dogs' heart rate was increased in both groups without any significant difference $(\mathrm{P}>0.05)$; however, the respiration rate in the experimental group was reduced significantly $(\mathrm{P}<0.05)$. It has to be mentioned that two dogs suffered apnea during the injection of fentanyl and were returned to normal respiration after 2-3 minutes by CPR. A significant different $(p<0.05)$ was observed in rectal temperature of the experiment group between first 10-minte-interval-period and last 10-minte-interval-period; moreover, a significant increase $(\mathrm{P}<0.05)$ was observed in the duration of analgesia and anesthesia. In this study, it was demonstrated that adding $0.1 \mathrm{mg} \mathrm{kg}$ of fentanyl to acepromazine and ketamine has a significant influence on the reduction of respiratory rate and the increase in the duration of analgesia and anesthesia; however, this addition does not have any significant influence on clinical symptoms of the dogs. Based on the results of this study, it can be suggested that this combination can be used as beneficial in the reduction of respiratory rate and the increase in the duration of analgesia and anesthesia.
\end{abstract}

KEYWORDS: Acepromazine; Dog; Fentanyl; General anesthesia; Ketamine

\section{INTRODUCTION}

Moderate anesthesia consists of soporific, relaxing and anesthetizing factors and for achieving the best result combinational protocol of multi-drug can be used. Ketamine $\mathrm{Hcl}$ or DL-2Chlorophenyl 2-Metil amin Cyclohexane Hydrochloride is a unique injective anesthetizing drug which first entered human medicine in the year 1965 and was first introduced as an anesthetizing drug for cats in the year 1970. This drug is acidic, and its acidity is between 3.5 to 5.5 . Immediately after the injection this drug is absorbed and distributed in all body tissues. The start of anesthetizing process is fast, but the throat reflexes will not vanish, and the rate of skeletal muscle will be increased in a way that it may be convulsive for dogs.
Quick Response Code:

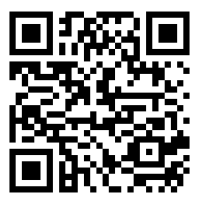

Address for correspondence: Mahmoud Tohidi Moghadam, Graduate of Faculty of Veterinary Medicine Islamic Azad University, Iran

Received: October 22, 2019 Published: November 20, 2019

How to cite this article: Adel Khodaei S, Mahmoud TM. Tips for Embryo Transfer Procedure in IVF/ICSI Cycle. 2019 - 1(3) OAJBS.ID.000114. DOI: 10.38125/OAJBS.000114 
This drug has a short-term and insignificant provocative effect on cardiovascular organs but sometimes it may have weakening effects on breathing. Also, it decreases vascular blood pressure in monkeys, but it increases it in dogs. In dogs it will cause increase of cardiovascular productivity and heart contraction power and decreases environmental resistance. In cats the start of anesthesia is after 3 to 5 minutes and anesthesia will last between 15 to 45 minutes and complete recovery from anesthesia will take 1 to 4 hours [1-3].

Fentanyl citrate is recognized with commercial names like Duragesic, Fundora, Instant l, Acted and Sublimize and is a derivative drug of Phenyl pyridine which is more fat-oriented that morphine $[4,5]$. This drug was first introduced by Paul Johnson in the year 1959 in newly established Johnson Pharmaceutical Laboratory. He performed evaluative tests on narcotic activities of patavine and succeeded to obtain the pharmaceutical structure of Fentanyl [6,7]. This drug is a complete agonist opioid with effect on Mo Kappa and Delta receivers. Anti-pain effect of Fentanyl in human is estimated as 100 times more than morphine and is considered as a drug with high level of mis consumption capability which is placed in the second pharmaceutical category [7].

Acepromazine Maleate is named structurally Acetyl promazine and is a derivative of phenothiazine and is highly used in veterinary medicine and can be applied in dogs, cats and horses according to FDA as a tranquilizer. Acepromazine is a yellowish, odorless, crystal powder drug with bitter taste. The powder of this drug will be melted in a temperature between 135 to 138 centigrade. Activities and applications of this drug is the same as its relatives, but the observing time of the symptoms is shorter in a way that tranquilizing effects can be observed in 10 minutes after consumption and 5 minutes after muscular injection [8].

Considering that today in injective anesthesia of dogs usually a combination of two or more drugs with anti-pain, anesthetizing, relaxing and tranquilizing effects simultaneously and considering that because using Fentanyl Citrate is not common among Iranian vets, thus the purpose of this study had been to evaluate the clinical characteristics of anesthesia caused by adding Fentanyl Citrate to combinational protocol of Ketamine- Acepromazine.

\section{MATERIALS AND METHODOLOGY}

Studied Animals: In this study 12 mixed-breed healthy dogs, that weighed 20-30 kg and are 2-3 years old were examined. The studied drugs in this research consisted on Acepromazine Maleate 2\%, manufactured by Kelle-Belgium Company, Ketamine Hydrochloride $10 \%$ manufactured by Alfas a Company-Netherlands and 500 mgr. Fentanyl Ampoule, manufactured by boogeyman Pharmaceutical Company-Iran.

12 hours before anesthesia are dogs were on special diet and they only had water to drink. After weighing the animals were put in the surgery room for 30 minutes to become familiar with the environment and then the path of cephalic in frontal body organs of the experimental dogs were sterilized and installed at its determined placed. These dogs were first subjected to muscular injection of combined protocol of Acepromazine and ketamine and after oneweek maintenance in equivalent nutritional and environmental condition they were subjected to injection of combined protocol of Acepromazine-Fentanyl-Ketamine.

Anesthesia in Control Group: Anesthesia using muscular injection of $0.2 \mathrm{mg}$ for each kilogram of Acepromazine and after 10 minutes and muscular injection of Ketamine in an amount of 20 $\mathrm{mg}$ for each kilogram of body weight was performed. Anesthesia in Experimental Group: Anesthesia using muscular injection of 0.2 mg for each kilogram of Acepromazine and after 10 minutes and vascular injection of 100 micrograms for each kilogram of fentanyl and immediate muscular injection of $20 \mathrm{mg}$ ketamine for each kilogram of body weight.

Measurement Method of the Studied Parameters: During one hour after anesthesia, in 10-minute intervals parameters such as heartbeat, breathe rate, body temperature, painful stimulation response and anesthesia duration were studied and registered. Any unexpected side effects such as convulsion, etc. (anesthesia report) were also registered in case of occurrence and the obtained data from each group were analyzed and compared. Evaluation of reacts toward painful stimulation by pressing the hand were also registered as presence or absence of any responses and pain level scaling as scaled as $+1,+2$ and +3 was avoided. The duration of anesthesia was also measured and registered as of the time of vanishing of head and neck writing reflexes till the time of its returning. Statistical Analysis of the Results: T test and variance analysis (ANOVA), and Tukey Post Hoc Test were used, and the meaningfulness of average differences were calculated based on $\mathrm{P}$ $<0.05$.

\section{RESULTS}

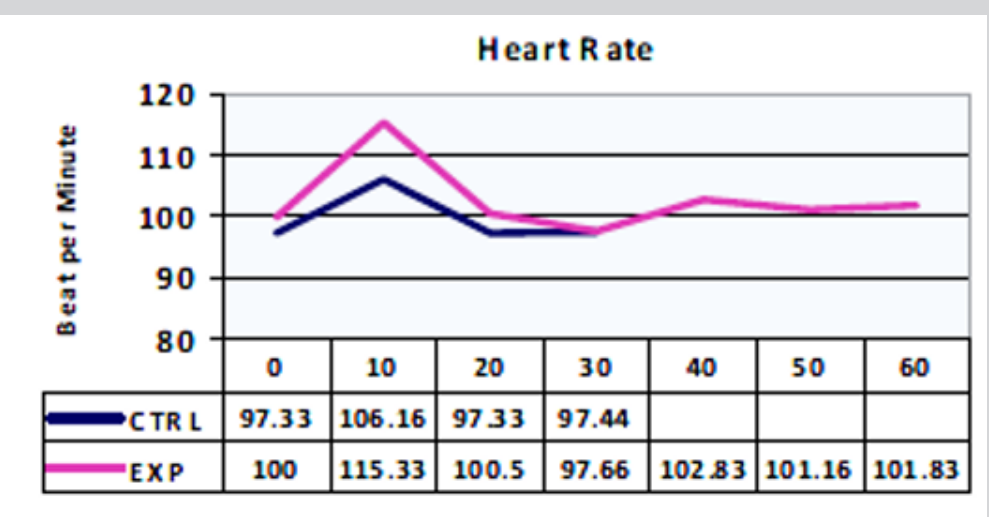

Time $S$ eries ( $m$ inute)

Figure 1: Heartbeat average changes in control \& experimental group at studied times. 


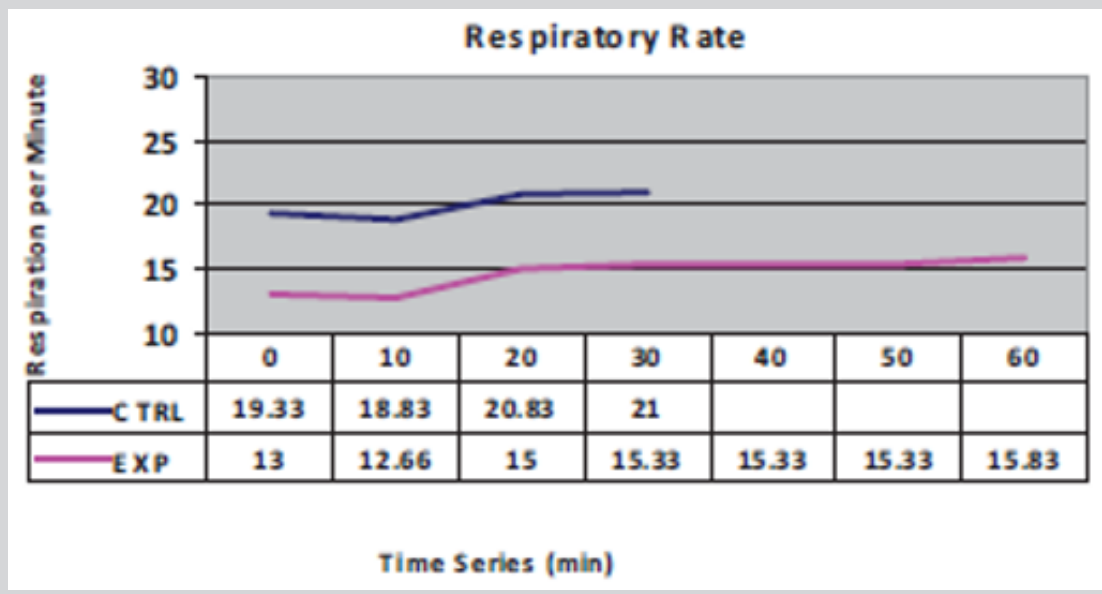

Figure 2: Breath rate average changes in control \& experimental group at studied times.

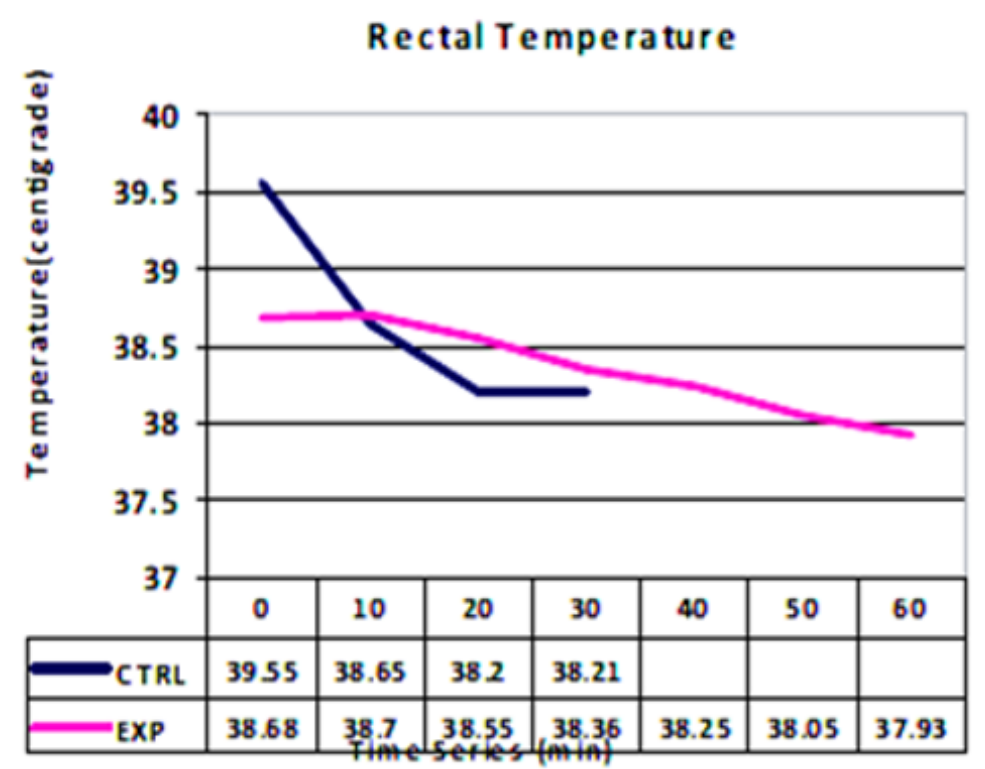

Figure 3: Rectal temperature average changes in control \& experimental group at studied times.

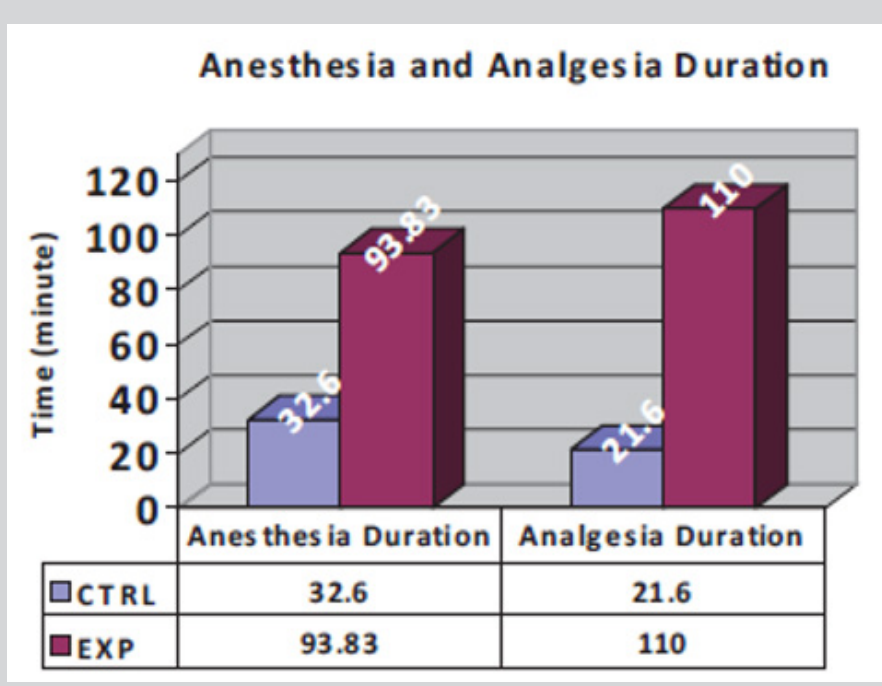

Figure 4: Comparison of anesthesia and numbness duration between control \& experimental group at studied times. 
Clinical results of the anesthesia caused by adding fentanyl to combination of ketamine and Acepromazine are indicated in Figures 1-4. It is worth mentioning that all of the dogs in the control group showed signs of returning from anesthesia before 40 minutes thus vital signs in this group were only measured and registered only till the $30^{\text {th }}$ minute after anesthesia but in the experimental group registration and measurement of the data continued till the $60^{\text {th }}$ minute after anesthesia. The process of changes in average heat beat, breath rate, body temperature, anesthesia and numbness duration and comparison of these quantities in control and experimental group at the studied times are respectively indicated in charts 1 to 4 .

\section{Statistical analysis results}

The results of T statistical test for the purpose of comparing the averages of heart beat, breath rate and rectal temperature shows the meaningful difference of $\mathrm{P}<0.05$ in comparing the average breath rate between control and experimental groups but with respect to heart beat and rectal temperature there is no meaningful difference observed between experimental and control groups (Table 1).

Table 1: Independent T-test for the purpose of comparing the average of heartbeat, breath rate and rectal temperature between experimental and control groups.

\begin{tabular}{|c|c|c|c|c|c|c|c|c|c|c|}
\hline \multicolumn{11}{|c|}{ Independent Samples Test } \\
\hline & & \multicolumn{2}{|c|}{$\begin{array}{l}\text { Levene's Test } \\
\text { for Equality } \\
\text { of Variances }\end{array}$} & \multicolumn{7}{|c|}{ t-test for Equality of Means } \\
\hline & & \multirow[t]{2}{*}{$\mathbf{F}$} & \multirow{2}{*}{ Sig. } & \multirow[t]{2}{*}{$\mathbf{t}$} & \multirow{2}{*}{ df } & \multirow{2}{*}{$\begin{array}{l}\text { Sig. (2 } \\
\text { tailed) }\end{array}$} & \multirow{2}{*}{$\begin{array}{c}\text { Mean } \\
\text { Difference }\end{array}$} & \multirow{2}{*}{$\begin{array}{l}\text { Std. Error } \\
\text { Difference }\end{array}$} & \multicolumn{2}{|c|}{$\begin{array}{c}\text { 95\% Confidence Interval } \\
\text { of the Difference }\end{array}$} \\
\hline & & & & & & & & & Lower & Upper \\
\hline \multirow{2}{*}{ Heart Rate } & $\begin{array}{l}\text { Equal valences } \\
\text { assumed }\end{array}$ & \multirow{2}{*}{0.091} & \multirow{2}{*}{0.764} & 0.264 & 58 & 0.793 & 2.48413 & 9.40755 & -16.34713 & 21.31538 \\
\hline & $\begin{array}{c}\text { Equal valences not } \\
\text { assumed }\end{array}$ & & & 0.266 & 32842 & 0.792 & 2.48413 & 9.33104 & -16.50349 & 21.47175 \\
\hline \multirow{2}{*}{$\begin{array}{l}\text { Respiratory } \\
\text { Rate }\end{array}$} & $\begin{array}{l}\text { Equal valences } \\
\text { assumed }\end{array}$ & \multirow{2}{*}{1.668} & \multirow{2}{*}{0.202} & -3.612 & 58 & 0.001 & -5.02381 & 1.39082 & -7.80784 & -2.23978 \\
\hline & $\begin{array}{c}\text { Equal valences not } \\
\text { assumed }\end{array}$ & & & -3.381 & 28.025 & 0.002 & -5.02381 & 1.48578 & -806718 & -1.98044 \\
\hline \multirow{2}{*}{ Temperature } & $\begin{array}{l}\text { Equal valences } \\
\text { assumed }\end{array}$ & \multirow{2}{*}{3.12} & \multirow{2}{*}{0.083} & -2.341 & 58 & 0.23 & -43810 & 0.18716 & -0.81274 & -0.6345 \\
\hline & $\begin{array}{c}\text { Equal valences not } \\
\text { assumed }\end{array}$ & & & -1.806 & 20.498 & 0.86 & -0.4381 & 0.24255 & -0.94325 & 0.6706 \\
\hline
\end{tabular}

The results of ANOVA statistical test for the purpose of studying the process of change in average heartbeat, breath rate and rectal temperature indicates a meaningful difference of $\mathrm{P}<0.05$ in the process of change in rectal temperature average at the studied time in the experimental group in a way that the results of Tukey Post
Hoc Test shows the meaningful difference in rectal temperature at the beginning of anesthesia and 10 minutes after than with 60 minutes after anesthesia. There is no meaningful difference observed in changes in averages of heart beats and breath rate at the studied times in the experimental group (Table 2).

Table 2: Variance Analysis Statistical Test (ANOVA) and Tukey Post Hoc Test were used for analysis of the changes in average heartbeat, breath rate and rectal temperature at the studied times in the experimental group.

\begin{tabular}{|c|c|c|c|c|c|c|c|c|}
\hline \multicolumn{9}{|c|}{ Descriptives } \\
\hline & \multirow{2}{*}{$\mathbf{N}$} & \multirow{2}{*}{ Mean } & \multirow{2}{*}{ Stl. Deviation } & \multirow{2}{*}{ Std. Error } & \multicolumn{2}{|c|}{ 95\% Confidence Interval for Mean } & \multirow{2}{*}{ Minimum } & \multirow{2}{*}{ Maximum } \\
\hline & & & & & Lower Bound & Upper Bound & & \\
\hline $\begin{array}{c}\text { Heart Rate } \\
\text { Base Line }\end{array}$ & 6 & 100 & 55.31004 & 22.58023 & 41.9557 & 158.0443 & 48 & 200 \\
\hline $10 \mathrm{~min}$ & 6 & 115.3333 & 50.74906 & 20.71822 & 62.0755 & 168.5912 & 68 & 200 \\
\hline $20 \mathrm{~min}$ & 6 & 100.5 & 31.0725 & 12.68529 & 67.8914 & 133.1086 & 72 & 140 \\
\hline $30 \mathrm{~min}$ & 6 & 97.6667 & 22.57137 & 9.21472 & 73.9795 & 121.3539 & 80 & 140 \\
\hline $40 \mathrm{~min}$ & 6 & 102.8333 & 24.50646 & 10.00472 & 77.1154 & 128.5513 & 76 & 144 \\
\hline $50 \mathrm{~min}$ & 6 & 101.1667 & 25.78695 & 10.52748 & 74.1049 & 128.2284 & 76 & 150 \\
\hline $60 \mathrm{~min}$ & 6 & 101.8333 & 25.20648 & 10.2905 & 75.3808 & 128.2859 & 80 & 150 \\
\hline Total & 42 & 102.7619 & 33.58775 & 5.1827 & 92.2952 & 113.2286 & 48 & 200 \\
\hline
\end{tabular}




\begin{tabular}{|c|c|c|c|c|c|c|c|c|}
\hline $\begin{array}{c}\text { Respiratory } \\
\text { Rate } \\
\text { Base Line }\end{array}$ & 6 & 13 & 3.52136 & 1.43759 & 9.3046 & 16.6954 & 10 & 20 \\
\hline $10 \mathrm{~min}$ & 6 & 12.6667 & 6.53197 & 2.66667 & 5.8118 & 19.5216 & 6 & 24 \\
\hline $20 \mathrm{~min}$ & 6 & 15 & 6.63325 & 2.70801 & 8.0388 & 21.9612 & 10 & 24 \\
\hline $30 \mathrm{~min}$ & 6 & 15.3333 & 4.36654 & 1.78263 & 10.7509 & 19.9157 & 12 & 23 \\
\hline $40 \mathrm{~min}$ & 6 & 15.3333 & 4.58984 & 1.8738 & 10.5166 & 20.1501 & 10 & 23 \\
\hline 50 min & 6 & 15.3333 & 3.61478 & 1.47573 & 11.5398 & 19.1268 & 11 & 21 \\
\hline 60 min & 6 & 15.8333 & 3.97073 & 1.62104 & 11.6663 & 20.0004 & 11 & 22 \\
\hline Total & 42 & 14.6429 & 4.67907 & 0.722 & 13.1848 & 38.101 & 6 & 24 \\
\hline Temperature & 6 & 38.6833 & 0.27869 & 0.11377 & 38.3909 & 38.9758 & 38.2 & 38.9 \\
\hline Base Line & 6 & 38.7 & 0.28983 & 0.11832 & 38.3958 & 39.0042 & 38.2 & 38.9 \\
\hline 10 min & 6 & 0.36194 & 0.14776 & 38.1702 & 38.9298 & 38.1 & 38.9 \\
\hline 20 min & 6 & 38.55 & 0.34448 & 0.14063 & 38.0052 & 38.7282 & 38 & 38.9 \\
\hline 30 min & 6 & 38.3667 & 0.42778 & 0.17464 & 37.8011 & 38.6989 & 37.7 & 38.9 \\
\hline 40 min & 6 & 38.25 & 0.54314 & 0.22174 & 37.48 & 38.62 & 37.3 & 38.9 \\
\hline 50 min & 6 & 38.05 & 0.55015 & 0.2246 & 37.356 & 38.5107 & 37.2 & 38.8 \\
\hline 60 min & 6 & 37.9333 & 0.4747 & 0.07325 & 38.214 & 38.5098 & 37.2 & 38.9 \\
\hline Total & 42 & 38.3619 & & & & & \\
\hline
\end{tabular}

\begin{tabular}{|c|c|c|c|c|c|c|}
\hline \multicolumn{7}{|c|}{ ANOVA } \\
\hline & & Sum of Squares & df & Mean Square & $\mathbf{F}$ & Sig. \\
\hline Heart Rate & Between Groups & 1200.952 & 6 & 200.159 & 0.155 & 0.987 \\
\hline & Within Groups & 45052.667 & 35 & 1287.219 & & \\
\hline & Total & 46253.619 & 41 & & & \\
\hline Respiratory Rate & Between Groups & 57.476 & 6 & 9.579 & 0.399 & 0.875 \\
\hline & Within Groups & 840.167 & 35 & 24.005 & & \\
\hline & Total & 897.643 & 41 & & & \\
\hline Temperature & Between Groups & 3.279 & 6 & 0.547 & 3.209 & 0.013 \\
\hline & Within Groups & 5.96 & 35 & 0.17 & & \\
\hline & Total & 9.239 & 41 & & & \\
\hline
\end{tabular}

The results of statistical T-test used for comparing the averages of duration of anesthesia period and numbness in control and experimental groups indicated a significant difference $(\mathrm{P}<0.05)$ in comparison of average of anesthesia period and numbness between control and experimental groups in a way that duration of anesthesia and numbness in experimental group is significantly greater in comparison with the control group (Table 3).

Table 3: Independent T-Test for Comparing average duration of anesthesia and numbness periods between control \& experimental groups.

\begin{tabular}{|c|c|c|c|c|c|c|c|c|c|}
\hline \multicolumn{10}{|c|}{ Independent Samples Test } \\
\hline & \multicolumn{2}{|c|}{\begin{tabular}{|c|} 
Levene's Test for \\
Equality of Variances
\end{tabular}} & \multicolumn{7}{|c|}{ t-test for Equality of Means } \\
\hline & \multirow[t]{2}{*}{$\mathbf{F}$} & \multirow[t]{2}{*}{ Sig. } & \multirow[t]{2}{*}{$t$} & \multirow[t]{2}{*}{ df } & \multirow[t]{2}{*}{$\begin{array}{l}\text { Sig. } \\
\text { (2-tailed) }\end{array}$} & \multirow[t]{2}{*}{$\begin{array}{c}\text { Mean } \\
\text { Difference }\end{array}$} & \multirow[t]{2}{*}{$\begin{array}{l}\text { Std. Error } \\
\text { Difference }\end{array}$} & \multicolumn{2}{|c|}{$\begin{array}{l}\text { 95\% Confidence Interval for } \\
\text { Mean }\end{array}$} \\
\hline & & & & & & & & Lower Bound & Upper Bound \\
\hline $\begin{array}{l}\text { Anesthesia Duration } \\
\text { Equal variances } \\
\text { assumed }\end{array}$ & \multirow[t]{2}{*}{3.888} & \multirow[t]{2}{*}{0.077} & 6.253 & 10 & 0 & 61.16667 & 9.78122 & 39.37276 & 82.96058 \\
\hline $\begin{array}{c}\text { Equal variances not } \\
\text { assured }\end{array}$ & & & 6.253 & 5.951 & 0.001 & 61.16667 & 9.78122 & 37.18499 & 85.14834 \\
\hline $\begin{array}{c}\text { Analgesia Duration } \\
\text { Equal variances } \\
\text { assured }\end{array}$ & 9.091 & \multirow[t]{2}{*}{0.013} & 8.318 & 10 & 0 & 88.31233 & 10.61969 & 64.67119 & 11199547 \\
\hline $\begin{array}{c}\text { Equal variances not } \\
\text { assured }\end{array}$ & & & 8.318 & 5.24 & 0 & 88.31233 & 10.61969 & 61.42439 & 11524228 \\
\hline
\end{tabular}




\section{DISCUSSION AND CONCLUSION}

Using general anesthesia technique for different types of diagnosis and surgery methods is necessary. Although sometimes bad incidents will happen during anesthesia, but we can obtain a pre-designed guaranteed method for appropriate anesthesia depth and creating appropriate and stable conditions for the patient during anesthesia and surgery operation and easy and safe recovery [2].

\section{HEARTBEAT RATE}

Using Ketamine drug will result in an increase in cardiovascular outputs, average aorta pressure, pulmonary veins pressure, vascular central pressure and heartbeat rate because of stimulating sympatric nerves [3]. Acepromazine will also decrease blood pressure by affecting vague nerves and blocking alpha-adrenergic receiver but in continuing it is possible that the body would react in response to this change and pressure decrease [1,2]. Fentanyl will decrease the heartbeat rate by affecting the mu receivers and by stimulating the vague nerve. He time and intensity depend on the injective dose and plasma concentration of the variable matter [8].

Considering that Fentanyl is a drug with high effective power and high speed result it should have had made this effect immediately in the animal but practically by vascular injection of 500 micrograms of Fentanyl Citrate no decrease was observed in heart beat rate in experimental group dogs during the anesthesia period and no meaningful difference was created between control and experimental groups.

In the year Farer [8] studied using Acepromazine in $0.2 \mathrm{mg}$ dose for each kilogram and ketamine in $10 \mathrm{mg}$ dose for each $\mathrm{kg}$ among 13 studied dogs. The results indicated that injecting Acepromazine will meaningfully decrease the blood pressure and as a result injecting Sc ketamine will also meaningfully increase heartbeat rate. In a research made by Mendes [2] on combination of Propofol, Fentanyl, AL Fentanyl or Fentanyl for inside vascular complete anesthesia on cats, decrease of heartbeat, blood pressure, rectal temperature and breath rate were observed in all the treatments [9]. Also, Ilia performed a research on cardiovascular effects of high dose of narcotics along with low dose of anesthesia inhaling drug in comparison with inhaling drug alone in dogs. In this study when the prescribed plasmatic concentration would reach 71.7 nanograms in each $\mathrm{ml}$, the quantity of MAC in loran decreased to 1.2 and heartbeat decreased meaningfully [10]. Also, during a research made by Rita in 1978 the effect of fentanyl with 5 microgram dose for each $\mathrm{kg}$ during the anesthesia period of Halothane gas on the heartbeat of dogs showed that using fentanyl with this dose will result in $10 \%$ decrease in heart beat rate during one hour study. Rita interpreted that approximately $90 \%$ of this state of fentanyl is because of increase in vagal efferent traffic from central neural system whereas only 10 and of it is as a result of weakening the sympathetic nerve tone [11]. Also, Loeb indicated that fentanyl can create an approximately individual activity on cardio vagal fibers which will result in change in heartbeat rate [12]

\section{Breath rate}

Comparing breath rate between control and experimental groups indicated decrease of breath rate in experimental group and meaningful difference between the two experimental and control groups. Ketamine muscular injection ordinarily does not have any effect on respiratory depression but in high doses and especially with vascular injection it is possible to observe this state in dogs [2]. Acepromazine has minor effects on breathing. Even it is possible that it may decrease the breathing rate a bit, but the volume of breathing is generally constant [2] and generally muscular injection of Acepromazine cannot create intense decrease in breathing during anesthesia. Thus, it seems that decrease in breath rate among dogs of experimental group is via direct effect of fentanyl drug on breathing control center in the brain stem which is created by being inducted on opioid receivers from mu type $[1,8]$. MU receivers are of two types, mu 1 receivers that will be resulted in numbness after being stimulated and $\mathrm{m} 2$ which will result in an increase in vascular expansion, decrease of heart beat rate and breathing rhythm and the intensity of stimulation of this receiver and its consequent results will be subjected and dependent to the applied dose [1,8]. Thus, in the present study decrease of breathing rate in experimental group and meaningful difference between the two experimental and control groups is resulted by injection of fentanyl and its immediate effect on breathing center. During a research performed by Bailey [14] on fentanyl and its effect on dog's anesthesia in different doses of 125, 500, 750, 1000, 1500, 2000 and 3000 micrograms for each kg using unit-dose and vascular method. All the doses resulted in a significant decrease in heartbeat rate and breathing rhythm but none of the mentioned quantities resulted in producing apnea or increasing $\mathrm{PaCO}_{2}$ to a level higher than $67 \mathrm{~mm} \mathrm{Hg}$ [13].

Stamen studied 40 young and healthy volunteers for studying the breathing states after the effect of fentanyl and alfentanil in human with 1.5 and 3 micrograms doses of fentanyl for each $\mathrm{kg}$ and alfentanil of 7.5 and 15 micrograms doses for each $\mathrm{kg}$ and the results showed that low and high doses of fentanyl will meaningfully decrease the breathing rate respectively till 30 minutes and 80 minutes after the injection whereas no respiratory depression was created in low dose of alfentanil and only high dose of it resulted in a decrease in the breathing rate during 4 minutes after injection [14].

According to the research made by Tanaka the effect of fentanyl along with propofol on breathing rate and heartbeat in 30 women patients subjected to vertebral column surgery were studied. In this study the patients were divided into 3 groups. FP group received propofol with $0.5 \mathrm{mg}$ dose for each $\mathrm{kg}$ along with 2 micrograms fentanyl for each kg. Group P received propofol along with normal saline and group $\mathrm{F}$ only received 2 micrograms fentanyl for each $\mathrm{kg}$. The results achieved in FP group showed higher decrease in breath rate and $\mathrm{CO}_{2}$ level in blood in comparison with the other 25 groups. In F and FP groups apnea production was also observed [15]. In the present study also immediate injection of fentanyl in 2 of the dogs resulted in an immediate decrease in breathing rate and apnea at the first minute that the animals automatically recovered to their ordinary breathing state by respiratory rehabilitation in approximately 2 minutes.

Rectal temperature: The results of comparing changes in temperature between the two control and experimental groups indicated that there is no meaningful difference between the two groups but in the experimental group there is a meaningful difference in temperature decrease between $0^{\text {th }}$ to $10^{\text {th }}$ minutes and the $60^{\text {th }}$ minute. In a research made by Limens in the year 2008 on Infusion fentanyl 5-10 micrograms for each $\mathrm{kg}$ per hour along with Acepromazine and Glycopyrrolate in dogs a meaningful decrease was observed in heart beat rate and body central temperature which was in conform to the obtained data from the experimental group in the present study [16]. 


\section{Anesthesia duration}

According to the averages achieved from the results, duration of anesthesia in experimental group was 93.83 minutes and in control group was 32.6 minutes which statistically indicates a meaningful difference between the experimental and control groups and it seems that using fentanyl drug will result in an increase in the anesthesia duration more that approximately 3 times in comparison of the control group which means that the need to anesthesia drug has meaningfully decreased.

During a research made by Ubicomp and Morrison on the effect of midazolam or fentanyl on the required dose of propofol in dogs, fentanyl significantly decreased the required dose of propofol in comparison with midazolam [6]. In the performed research by Keriado also in studying the decrease of MAC isoflurane using fentanyl and remifentanil which was performed on rats, fentanyl group with 15, 40 and 60 micrograms doses for each kg per hour and remifentanil with 60,120 and 240 micrograms doses for each kg per hour was challenged sing continuous vascular infusion method and the quantity of MAC isoflurane in all groups similarly decreased $10 \%$ in low doses, $25 \%$ in medium doses and $60 \%$ in high doses [17].

\section{NUMBNESS DURATION}

Average period of numbness in experimental group were 110 minutes and in control group was 21.6 minutes that shows that there is a completely meaningful difference between the experimental and control groups. The results achieved from the studies made by Van Den Hogan show that in comparing numbness caused by injection of epidural and morphine, meperidine (Pethidine), Fentanyl and Fentanyl in rats show that rats that received fentanyl with $0.16 \mathrm{mg} /$ $\mathrm{kg}$ dose had a peak consciousness time approximately between 118 to 119 minutes and numbness duration was calculated as approximately 150 minutes [18].

During the performed research made by Waterman with respect to evaluation of numbness level created by fentanyl in sheep using thermal and mechanical tests, vascular injection of 5 micrograms fentanyl created approximately 30 minutes of numbness against thermal stimulators but it did not show an appropriate numbness activity with respect to mechanical stimulators. In comparison of 10 micrograms for each $\mathrm{kg}$ dose of vascular injection it created 60 minutes of thermal numbness and 40 minutes of numbness against mechanical test. Moreover, in one of the sheep with 20 micrograms for each $\mathrm{kg}$ dose a thermal numbness of 110 minutes and mechanical numbness of 60 minutes were created that all show that the level and duration of numbness with fentanyl depends on the applied dose [19]. In the present study also by injecting $0.1 \mathrm{mg}$ fentanyl for each $\mathrm{kg}$, average duration of numbness of 110 minutes was achieved against mechanical stimulators.

Generally in evaluating the weak and strong points of adding fentanyl to the combined protocol of Acepromazine-Ketamine we can conclude that slow vascular injection of $0.1 \mathrm{mg}$ dose for each $\mathrm{kg}$ in dogs had no negative and decreasing effect on the hearts muscle and created appropriate duration of numbness and anesthesia and in contrast will cause decrease of breathing rate and creation of minor hypothermia. Thus, in using this combined protocol we can have an appropriate anesthesia in dog by providing monitoring and confronting approaches for respiratory depression, apnea and hypothermia.

\section{APPRECIATION}

The writers appreciate the cooperation of Abdurrahman Pharmaceutical Company in presenting fentanyl drug and scientific and technical cooperation of Razak Laboratories Pharmaceutical Company.

\section{REFERENCES}

1. Brander GC, Pugh DM, Bywater RJ (1992) Veterinary applied pharmacology and therapeutics Billier Tindall.

2. Mendes GM, Selimi AL (2003) Use of combination of propofol and fentanyl, alfentanil or sufentanyl for total intravenous anesthesia in cats. J Am Vet Med Assoc 223(11): 1608-1613.

3. Sedighi MR (1999) Anesthesia in small animal. Ferdoci University.

4. 2003-2006 Center for drug evaluation and research (FDA) pharmacology/ Toxicology review and evaluation. NDA number 21(338): 1-75.

5. Chase PE (1977) Problem-oriented approach to anesthesia. Feline Pact 7: 24-26.

6. Corey CGL, Murison PJ (2008) Fentanyl or midazolam for co-induction of anesthesia with propofol in dogs. Vet Anaesth Analg 35(6): 463-472.

7. Stanley TH (1992) The history and development of the fentanyl series. J Pain Symptom Manage 7 (3): S3-S7.

8. Farver TB, Haskins SC, Patz JD (1986) Cardiopulmonary effects of acepromazine and of the subsequent administration of ketamine in the dog. Am J Vet Res 47(3): 631-635.

9. Alexander F (1998) Veterinary pharmacology ( $4^{\text {th }}$ edn), 245.

10. Reitan JA, Stengert KB, Wymore ML, Martucci RW (1978) Central vagal control of fentanyl induced bradycardia during halothane. Anesth Analg 57(1): 31-36.

11. Jerod M, Peter R, Lichtenthala, John M, Tarnowskya (1984) Parasympathomimetic effects of fentanyl on the canine sinus node. J Auton Nerv Syst 11(1): 91-94.

12. Satoru T, Hideaki T, Hajime S, Akiyoshi N (1998) Respiratory and cardiovascular effects of fentanyl during propofol-induced sedation under spinal anesthesia. J Anesth 12(4): 171-174.

13. Lumb WV, Jones EW (1973) Veterinary anesthesia. ( $2^{\text {nd }}$ edn), Lea \& febiger, Philadelphia, USA.

14. Bailey PL, Port JD, McJames S, Reinersman L, Stanley TH (1987) Is fentanyl an anesthetic in the dog? Anesth Analg 66(6): 542-548.

15. Scumman Fl, Ghoneim MM, Korttila K (1984) Veterinary and mental effects of alfentanil and fentanyl. Act Anaesthesiol Scand 28(1): 63-67.

16. Susanne l, Peter JS (2008) The cardiorespiratory effects of a fentanyl infusion following acepromazine and glycopyrrolate in dogs. Tijdschr Diergeneeskd 133(21): 888-895.

17. Ilkiw JE, Pascoe PJ, Haskins SC, Patz JD, Jaffe R (1994) The cardiovascular sparing effect of fentanyl and atropine administered to enflurane anesthetized dogs. Can J Vet Res 58(4): 248-253.

18. Criado AB, Gómez IAS (2003) Reduction of isoflurane MAC by fentanyl or remifentanil in rats. Vet Anaesth Analog 30(4): 250-256.

19. Van den Hoogen RH, Colpaert FC (1987) Epidural and subcutaneous morphine, meperidine (pethidine), fentanyl and sufentanil in the rat: analgesia and other invivo pharmacology effects. Anesthesiology 66(2): 186-194.

20. Waterman AE, Livingston A, Amin A (1990) The antinociceptive activity and respiratory effects of fentanyl in sheep. Veterinary Anesthesia and Analgesia 17(1): 20-23. 